although survival has improved over recent years, morbidity remains high because of both the disease process itself and the toxicity associated with therapy. Although the underlying cause of SLE is unknown, animal studies suggest that $B$ cells could have a key role in pathogenesis. Rituximab is a chimeric anti-CD20 antibody that reduces $\mathrm{B}$-cell numbers in vivo and has been used to treat lymphoproliferative diseases in children and adults. Emerging data suggests it might also be efficacious in treating autoimmune diseases.

In seven children with SLE that was refractive to conventional immunosuppression, treatment with two $750 \mathrm{mg} / \mathrm{m}^{2}$ intravenous infusions of rituximab given over 2 weeks with high-dose corticosteroids \pm cyclophosphamide resulted in significant improvements in British Isles Lupus Assessment Group global scores from a median of 22 at baseline to 6 at followup (median follow-up 1 year; $P=0.002$ ). Improvements were seen in all clinical symptoms which had indicated treatment, including two cases of life-threatening disease that did not respond to conventional therapy. No significant adverse events were reported in this group of children.

B-lymphocyte depletion using rituximab in combination with immunosuppressive therapy was therefore a safe and effective treatment in this small patient cohort and could be a useful treatment for children with SLE which is refractory to conventional therapy. Multicenter, randomized clinical trials of rituximab are now required to confirm these results.

Carol Lovegrove

Original article Marks SD et al. (2005) B lymphocyte depletion therapy in children with refractory systemic lupus erythematosis. Arthritis Rheum 52: 3168-3174

\section{Smoking and thrombotic events in SLE}

Smoking and disease activity are strongly associated with thrombotic events in patients with systemic lupus erythematosus (SLE), according to a recent report of data from the LUMINA (Lupus in Minorities: Nature versus Nurture) patient cohort.

A number of risk factors and disease-related factors might be linked to thrombotic events in patients with SLE; of these, an association with the presence of antiphospholipid antibodies (aPL) has been demonstrated. A number of trials have recommended the use of hydroxychloroquine, known to have immunosuppressant and antiplatelet activity, as thromboembolic prophylaxis in patients with ANTIPHOSPHOLIPID SYNDROME.

To determine the relationship between the presence of aPL, hydroxychloroquine use and the occurrence of thrombotic events (arterial and/or venous; peripheral and/or central), Ho and colleagues studied 442 patients with SLE by generalized estimating equation (GEE). Although a number of variables were significant in univariate analysis, only smoking and disease activity remained significant by multivariate GEE analysis: aPL were not significant in either analysis, and although hydroxychloroquine use was protective in univariate analysis, this significance was not maintained in multivariate GEE.

Although disease activity is generally efficiently managed, these data emphasize that overall management should also include encouragement of patients with SLE to stop smoking in order to reduce their risk of thrombotic events.

Carol Lovegrove

Original article Ho KT et al. (2005) Systemic lupus erythematosus in a multiethnic cohort (LUMINA): XXVIII. Factors predictive of thrombotic events. Rheumatology 44 : 1303-1307

\section{Use of alendronate to prevent femoral-head collapse}

Osteonecrosis of the femoral head is responsible for a large proportion of primary total hip replacements. Collapse of the femoral head is thought to be a consequence of an uncoupling of the rates of osteoclastic bone resorption and osteoblastic bone regeneration; however, there is no universally accepted treatment for the prevention of collapse. Lai et al. have found that treatment with alendronate seems to prevent early collapse.

A total of 40 patients were randomly allocated to either the alendronate or no-treatment (control) group and followed for between 24 and 28 months. Inclusion criteria were stage II or III nontraumatic osteonecrosis of one or both femoral heads according to STEINBERG CLASSIFICATION, necrotic area of $>30 \%$ (class C

\section{GLOSSARY}

ANTIPHOSPHOLIPID SYNDROME

A clinical syndrome of thrombosis (arterial or venous) and/or recurrent miscarriage in the presence of antiphospholipid antibodies and/or the lupus anticoagulant

\section{STEINBERG}

CLASSIFICATION

A system of classification for osteonecrosis of the femoral head, with stages $0-\mathrm{VI}$ describing increasingly severe levels of radiographic damage 